\title{
Estado nutricional, características de la vivienda y crianza de animales de traspatio como factores asociados a enteroparasitosis en niños
}

\author{
Nutritional status, characteristics of housing and breeding of backyard \\ animals as factors associated with enteric parasites in children \\ David Henry Serrano Ramos ${ }^{1}$, Aldo Alim Valderrama Pomé ${ }^{1,2}$
}

\section{Resumen}

El objetivo del estudio fue determinar la asociación de la enteroparasitosis en niños en edad escolar del distrito de Santa María de Chicmo (Apurímac, Perú) con el estado nutricional, características de la vivienda y crianza de animales de traspatio. Se tomaron muestras coprológicas a 493 niños de 6-16 años, de ambos sexos y se visitaron sus viviendas para determinar sus características. La prevalencia de enteroparásitos fue de $82.4 \%$, donde la mayor prevalencia correspondió a Entamoeba coli $(59.6 \%)$, seguida de Ascaris lumbricoides (51.1\%) y Taenia sp con 33.3\%. Otros parásitos presentaron prevalencias menores a $12 \%$. La crianza de porcinos y pollos estuvo asociada a Taenia sp y E. vermicularis, respectivamente. Defecar a campo abierto, vivienda al borde de cultivos y al borde de acequias estuvieron asociados a $E$. vermicularis, $H$. nana y $A$. lumbricoides. El estado nutricional no estuvo asociado a la parasitosis en los escolares. Se concluye que las características de las viviendas y la crianza de animales de traspatio presentan características que favorecen la presentación de parasitismo intestinal en los niños del distrito de Santa María de Chicmo.

Palabras clave: parásitos, niño, estado nutricional, vivienda, animales de traspatio

\footnotetext{
${ }^{1}$ Facultad de Medicina Veterinaria y Zootecnia, Universidad Nacional Micaela Bastidas de Apurímac - UNAMBA, Abancay, Perú

${ }^{2}$ E-mail: alimvalderrama@gmail.com

El artículo es derivado del trabajo de tesis de David Henry Serrano Ramos para obtener el grado de Médico Veterinario y Zootecnista en la Universidad Nacional Micaela Bastidas de Apurímac, Perú
} 
The aim of this study was to determine the association of enteroparasitosis in school-age children in the Santa María de Chicmo district (Apurímac, Peru) with the nutritional status, characteristics of the house, and raising of backyard animals. Stool samples were taken from 493 children aged 6-16 years, of both sexes, and their homes were visited to determine their characteristics. The prevalence of enteroparasites was $82.4 \%$, where the highest prevalence corresponded to Entamoeba coli $(59.6 \%)$, followed by Ascaris lumbricoides (51.1\%) and Taenia sp with 33.3\%. Other parasites presented prevalence less than $12 \%$. The raising of pigs and chickens was associated with Taenia sp and E. vermicularis, respectively. Defecating in the open field, housing on the edge of crops and on the edge of ditches were associated with E. vermicularis, H. nana and $A$. lumbricoides. Nutritional status was not associated with parasitosis in school children. It is concluded that the characteristics of the houses and the raising of backyard animals present characteristics that favour the presentation of intestinal parasitism in children in the Santa María de Chicmo district.

Key words: parasites, children, nutritional status, living place, backyard animals

\section{INTRODUCCIÓN}

Los niños de edad escolar son un grupo de riesgo vulnerable para adquirir infecciones parasitarias. Así mismo, una vez que un niño es infectado, la probabilidad de contagio hacia sus familiares cercanos es alta (Iannacone et al., 2006; Steinmann et al., 2010). Las parasitosis intestinales son un problema de importancia al que se enfrentan las instituciones de salud pública y ambiental en países en vías de desarrollo (Solano et al., 2008). En Perú, las infecciones por protozoarios y helmintos intestinales presentan elevadas prevalencias (Ibáñez et al., 2004), donde $30 \%$ de niños entre 2 y 10 años presentan algún tipo de endoparasitosis (Altamirano et al., 2014).

Las infecciones parasitarias son generalmente subestimadas por ser asintomáticas, pero representan un factor de morbilidad importante cuando se asocian a la desnutrición. Las enteroparasitosis pueden transcurrir asintomáticas durante largo tiempo, pero tam- bién pueden llegar a provocar cuadros digestivos, inclusive con severa repercusión sobre el crecimiento y desarrollo de los niños (Solano et al., 2008). Las deficientes condiciones sanitarias predisponen a un mayor riesgo de infección por helmintos y protozoarios, lo cual repercute en su estado nutricional (Iannacone et al., 2006).

Existe una serie de factores socioeconómicos que favorece el mantenimiento de las infecciones parasitarias, tales como los relacionados a la vivienda, como son el tipo de construcción, hacinamiento y disponibilidad de servicios básicos (agua y desagüe), entre otros (Altamirano et al., 2014). En el distrito de Santa María de Chicmo, Apurímac, Perú, no existen estudios al respecto, a pesar de que presenta características ambientales y sociales similares a las zonas hiperendémicas de Perú. En consecuencia, el objetivo de esta investigación fue determinar la asociación entre enteroparasitosis con el estado nutricional, características de la vivienda y crianza de animales de traspatio del distrito. 
Cuadro 1. Características demográficas de los niños considerados en el estudio

\begin{tabular}{lcc}
\hline $\begin{array}{l}\text { Características } \\
\text { demográficas }\end{array}$ & $\mathrm{n}$ & $\%$ \\
\hline $\begin{array}{c}\text { Grupo etario } \\
<12 \text { años }\end{array}$ & 290 & 58.8 \\
$\quad \geq 12$ años & 203 & 41.2 \\
Sexo & & \\
Femenino & 248 & 50.3 \\
Masculino & 245 & 49.7 \\
\hline Total & 493 & 100.0 \\
\hline
\end{tabular}

\section{MATERIALES y Métodos}

El estudio se realizó en Santa María de Chicmo, uno de los 19 distritos de la provincia de Andahuaylas, región Apurímac, Perú, ubicado a una altitud de $3272 \mathrm{~m}$. Cuenta con una superficie de $162.14 \mathrm{~km}^{2}$, con una población estimada de 9910 habitantes y $79.4 \%$ de pobreza, donde $84 \%$ de la población económicamente activa ejerce la agricultura y solo 288 de las 2478 viviendas del distrito tienen acceso a agua potable de la red pública (INEI, 2018). El clima es generalmente cálido y templado, con una temperatura media anual de $15.3{ }^{\circ} \mathrm{C}$. En comparación con el invierno, los veranos son más lluviosos, con una precipitación media de $930 \mathrm{~mm}$. La precipitación más baja es en julio $(10 \mathrm{~mm})$, mientras que en febrero alcanza $179 \mathrm{~mm}$ en promedio. Por otro lado, presenta alta humedad atmosférica (SENAMHI, 2018).

El estudio se realizó entre setiembre de 2016 y abril de 2017. Los análisis coprológicos se efectuaron en el Laboratorio Referencial de la Dirección Regional de Salud (DIRESA) de la ciudad de Abancay, Apurímac. El tamaño de muestra para determinar la prevalencia de enteroparasitosis, mediante un muestreo simple al azar, fue de 327 niños; sin embargo, se logró tomar 493 muestras de niños y niñas entre 6-16 años, repartidas proporcionalmente entre las 23 instituciones educativas del distrito (Cuadro 1). Las muestras se obtuvieron coordinando previamente con los directores de las instituciones educativas. Los niños incluidos en el estudio fueron elegidos aleatoriamente. A cada niño se le entregó un frasco para la toma de muestra de heces, una ficha para el consentimiento informado para ser llenada por el padre o apoderado y una ficha para el asentimiento del niño.

A cada niño se le aplicó una entrevista epidemiológica para identificar las características de su vivienda y la crianza de animales de traspatio; asimismo, se les tomó medidas antropométricas para determinar su estado nutricional. Los procedimientos de laboratorio se siguieron conforme a los protocolos del Instituto Nacional de Salud (Beltrán et al., 2003; Uribe et al., 2012) del Ministerio de Salud de Perú.

Las muestras de heces (3-6 g) fueron trasladadas al laboratorio de la DIRESA Apurímac en un tiempo de 2-4 h de su obtención (Beltrán et al., 2003). Se utilizó como prueba de tamizaje la técnica de sedimentación rápida (TSR) (concentración por sedimentación sin centrifugación) (Maco et al., 2002a; Beltrán et al., 2003; Uribe et al., 2012). Después, para todas las muestras que dieron positivo, se procedió con el método cuantitativo de Kato-Katz (análisis cuantitativo) el cual determina el número de huevos por gramo de heces (hpg) (Sánchez et al., 2010; Beltrán et al., 2003; Uribe et al., 2012). La cantidad de huevos hallados en la lámina se multiplicó por $\mathrm{k}(\mathrm{k}=24)$ para obtener el número de huevos por gramo de heces (hpg). En caso de deposiciones pastosas o líquidas se usaron los factores de corrección ( $\mathrm{k} / 2$ para deposiciones «sueltas» y k/3 para deposiciones diarreicas).

Las características de la vivienda y de la crianza de animales de traspatio se obtuvieron realizando una visita a las viviendas de los niños utilizando guías de observación. 
Para el caso de la vivienda se identificaron posibles factores asociados al parasitismo como suministro de agua por canales, tener piso de tierra, tener una habitación en la vivienda y ubicación al borde de acequia, canal de regadío o cultivos; así mismo, se registró si defecaban a campo abierto, si había letrinas y si había presencia de actividad agrícola. Para el caso de animales de traspatio se indagó sobre la crianza de bovinos, ovinos, perros, y otros.

La guía de observación para las características de la vivienda y crianza de animales fue un instrumento unidimensional desarrollado por los autores; para ello, se requirió el juicio de expertos, cuya especialización, experiencia profesional, académica e investigativa relacionada al tema a investigar facultó la evaluación de contenido y de forma de cada uno de los ítems considerados en la herramienta. Seguidamente, de acuerdo con los resultados del análisis de concordancia entre los jueces expertos, se procedió a una segunda redacción de ítems que conformaron el instrumento, con el cual se realizó una prueba piloto. Esta consideró las características de la población, similares a la muestra que se investigó posteriormente.

El estado nutricional de los niños se determinó a través del Índice de Masa Corporal (IMC), varones y mujeres por separado, de acuerdo con el protocolo del Centro Nacional de Alimentación y Nutrición de la Dirección Ejecutiva de Prevención de Riesgo y Daño Nutricional del Instituto Nacional de Salud de Perú (Aguilar et al., 2015). Con las estimaciones de peso y talla del niño se calculó el IMC $\left(\mathrm{kg} / \mathrm{m}^{2}\right)$, según la fórmula: $\mathrm{IMC}=$ peso $(\mathrm{kg}) /$ talla $(\mathrm{m})$. A los varones y mujeres de 10-16 años se les calculó la edad biológica con ayuda de los estadios de Tanner (Aguilar et al., 2015). Para ello, se mostró a los niños las ilustraciones de Tanner para que indiquen el estadio de desarrollo corporal en el que se encontraban. Si la edad cronológica y la edad biológica diferían en un año o más, se utilizó la edad biológica para valorar el estado nutricional según el IMC/Edad y Talla/Edad. En la realización de estas pruebas se contó con la presencia de la madre, padre o tutor anticipadamente informado, o de otro personal de salud de igual sexo que el niño evaluado.

Para determinar el peso de los niños se utilizó una balanza digital CA-MI, modelo SPRINT $( \pm 100 \mathrm{~g})$. Para medir la altura de los niños se utilizó el tallímetro mecánico de pared CHARDER HM201D ( $\pm 1 \mathrm{~mm})$. Una sola persona realizó las mediciones de talla, peso y las estimaciones de edad biológica para evitar el sesgo en la obtención de los valores.

El análisis de los datos se hizo con el programa SPSS v. 23. Se compararon las variables categóricas con el test de Chi cuadrado y el Odds ratio (OR) con intervalos de confianza al $95 \%$ y valor de $\mathrm{p}<0.05$ como nivel crítico de significancia. Asimismo, se realizó una prueba de regresión logística multivariada para determinar posibles asociaciones con la enteroparasitosis.

El proyecto de investigación fue aprobado por el Instituto Nacional de Salud, mediante Resolución Directoral № 870-2016OGITT-OPE/INS, el cual contempló el visto bueno de la Dirección Ejecutiva de Investigación de la OGITT del INS y la opinión favorable del Comité Institucional de Ética en Investigación del Instituto Nacional de Salud.

\section{Resultados}

La prevalencia de enteroparásitos en niños fue de $82.4 \%$ (407/493), donde la mayor prevalencia correspondió a Entamoeba coli con 59.6\% (294/493); seguida de Ascaris lumbricoides con 51.1\% (252/493) y Taenia sp con 33.3\% (164/493) (Cuadro 2). Cabe mencionar que la prevalencia de Enterobius vermicularis podría estar subestimada debido a que no se usó la técnica de la cinta adhesiva para la recuperación de huevos de piel anal y perianal (Test de Graham). 
Cuadro 2. Prevalencia de enteroparásitos en niños del distrito de Santa María de Chicmo, Apurímac, Perú

\begin{tabular}{lccc}
\hline Enteroparásitos & $\mathrm{n}$ & $\%$ & $\mathrm{IC}(95 \%)$ \\
\hline Entamoeba coli & 294 & 59.6 & $55.2-64.1$ \\
Ascaris lumbricoides & 252 & 51.1 & $46.6-55.6$ \\
Taenia sp & 164 & 33.3 & $29.0-37.5$ \\
Enterobius vermicularis & 58 & 11.8 & $8.8-14.7$ \\
Hymenolepis nana $^{1}$ & 36 & 7.3 & $4.9-9.7$ \\
Otros $^{1}$ & 34 & 6.9 & $4.6-9.2$ \\
\hline Total & 407 & 82.4 & $78.9-85.9$ \\
\hline
\end{tabular}

${ }^{1}$ Balantidium sp, Fasciola hepatica, Paragonimus sp, Trichuris trichiura, Ancylostoma $\mathrm{sp}$, Diphyllobothrium sp, Trichostrongylus sp

\section{Otros}

\section{A. lumbricoides}

H. nana

E. vernicularis

Taenia sp

E. coli

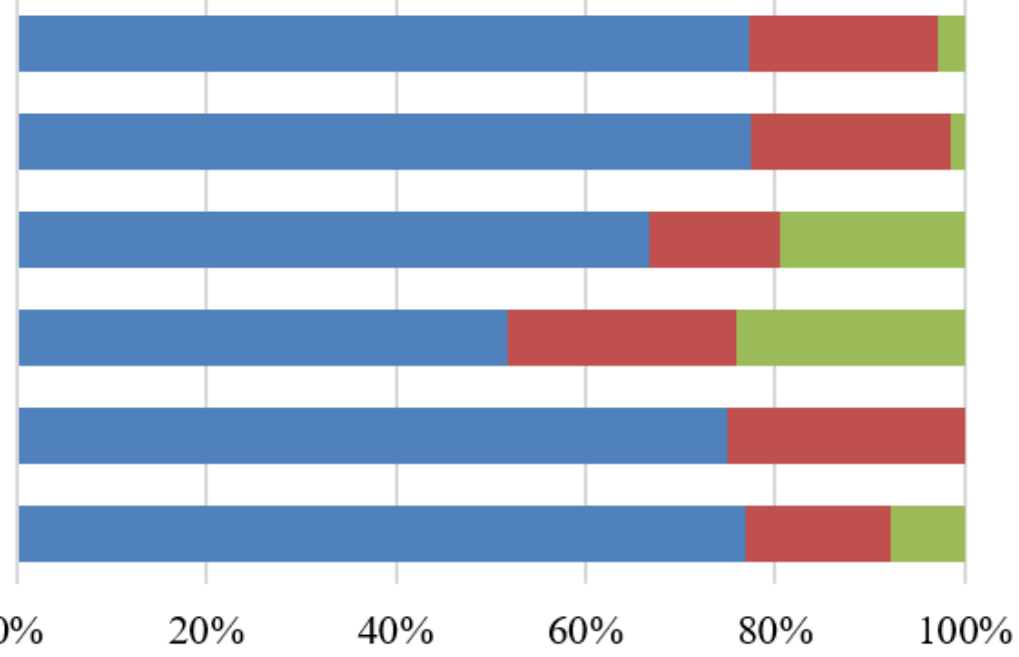

- Leve $\quad$ Moderada $\quad$ Severa

Figura 1. Intensidad de la infección por enteroparásitos en niños del distrito de Santa María de Chicmo, Apurímac, Perú, mediante el método cuantitativo de Kato-Katz

La Figura 1 muestra los resultados del análisis coprológico con el método de KatoKatz de las muestras positivas a la prueba de TSR, donde los enteroparásitos que presentaron intensidad severa de infección parasitaria en los niños fueron $E$. vermicularis con $24.1 \%(14 / 58 ;$ IC95\% $=12.3-36.0), H$. nana con $19.4 \%(7 / 36 ;$ IC95\% $=5.1-33.8)$ y $E$. coli con $7.8 \%(23 / 294 ;$ IC95\% $=4.6-11.1)$. Por otro lado, el $36.7 \%$ de los niños tuvieron biparasitismo (181/493; IC95\% = 32.4-41.1), $21.9 \%$ monoparasitismo (108/493; IC95\% = $18.2-25.7)$ y $24.3 \%$ poliparasitismo (120/493; IC95\% $=20.5-28.2)$. El $21.3 \%$ de los niños 
Cuadro 3. Características de las viviendas y de la crianza de animales de traspatio en los hogares de niños del distrito de Santa María de Chicmo, Apurímac, Perú

\begin{tabular}{|c|c|c|c|c|}
\hline Característica & & $\mathrm{n}$ & $\%$ & IC 95\% \\
\hline \multirow[t]{4}{*}{ Vivienda } & $\begin{array}{l}\text { Con disposición de } \\
\text { excretas en letrina }\end{array}$ & 468 & 94.9 & $92.9-96.9$ \\
\hline & $\begin{array}{l}\text { Con disposición de } \\
\text { excretas a campo } \\
\text { abierto }\end{array}$ & 454 & 92.1 & $89.6-94.6$ \\
\hline & $\begin{array}{l}\text { Con una sola } \\
\text { habitación }\end{array}$ & 422 & 85.6 & $82.4-88.8$ \\
\hline & Otros $^{1}$ & & $<80$ & \\
\hline \multirow[t]{4}{*}{ Animales } & Cuyes & 465 & 94.3 & $92.2-96.5$ \\
\hline & Porcinos & 421 & 85.4 & $82.2-88.6$ \\
\hline & Pollos & 401 & 81.3 & $77.8-84.9$ \\
\hline & Otros $^{2}$ & & $<80$ & \\
\hline
\end{tabular}

${ }^{1}$ Con piso de tierra; con suministro de agua por canales de regadío; al borde de acequia; al borde de canal de regadío o cultivos

2 Bovinos; ovinos; patos; perros; conejos

Cuadro 4. Factores asociados a enteroparasitosis en niños de Santa María de Chicmo (Apurímac, Perú), determinados por análisis de regresión logística multivariada

\begin{tabular}{|c|c|c|c|c|c|c|c|c|}
\hline Factor asociado & 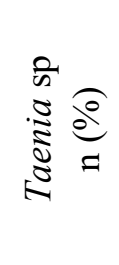 & OR & 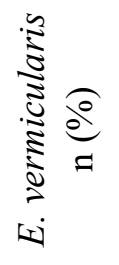 & OR & 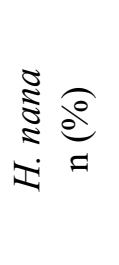 & OR & 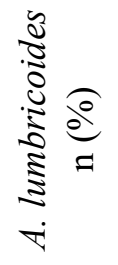 & OR \\
\hline $\begin{array}{l}\text { Defecar a campo } \\
\text { abierto }(n=454)\end{array}$ & & & $\begin{array}{c}{ }^{\mathrm{b}} 49 \\
(10.8)\end{array}$ & 2.9 & & & & \\
\hline $\begin{array}{l}\text { Vivir al borde de } \\
\text { cultivos }(n=94)\end{array}$ & & & & & $\begin{array}{c}{ }^{\mathrm{a}} 1 \\
(1.1)\end{array}$ & 8.9 & & \\
\hline $\begin{array}{l}\text { Vivir al borde de } \\
\text { acequias }(\mathrm{n}=132)\end{array}$ & & & & & & & $\begin{array}{c}\text { b59 } \\
(44.7)\end{array}$ & 2.1 \\
\hline $\begin{array}{l}\text { Crianza de } \\
\text { porcinos }(n=421)\end{array}$ & $\begin{array}{l}{ }^{\mathrm{a}} 150 \\
(35.6)\end{array}$ & 2.2 & & & & & & \\
\hline $\begin{array}{l}\text { Crianza de pollos } \\
(\mathrm{n}=401)\end{array}$ & & & $\begin{array}{c}{ }^{\mathrm{b}} 53 \\
(13.2)\end{array}$ & 3.8 & & & & \\
\hline
\end{tabular}

${ }^{a} p<0.05 ;{ }^{b} p<0.01$ 
presentaron parasitosis por protozoarios (105/493), $8.7 \%$ por helmintos (43/493) y $51.5 \%$ presentaron parasitosis mixta $(254 / 493)$.

El estado nutricional antropométrico resultó ser normal en $97.4 \%$ de los niños $(480 / 493 ;$ IC95\% $=95.9-98.9)$, con sobre peso en $1.8 \%(9 / 493$; IC95\% $=0.5-3.1)$ y con bajo peso en $0.8 \%(4 / 493$; IC95\% $=0.2-2.1)$.

Las características más relevantes de las viviendas fue que poseían suministro de agua por canales de regadío (93.5\%), presentaban actividad agrícola $(92.9 \%)$, tenían piso de tierra (83.2\%) y estaban ubicadas al borde de cultivos (80.9\%) (Cuadro 3). Los canales de regadío tienen la función de llevar agua a la huerta donde es aprovechada en los cultivos, por lo que no debería de servir para consumo humano. Por otro lado, en la mayoría de las viviendas se criaban animales (99.6\%), tales como cuyes, pollos, porcinos y perros (Cuadro 3)

El Cuadro 4 muestra los resultados del análisis de regresión logística multivariada, donde defecar a campo abierto estuvo asociado a la infección con $E$. vermicularis $(\mathrm{OR}=2.9 ; \mathrm{IC} 95 \%=1.3-6.7)$; vivir al borde de cultivos estuvo asociado a la infección con H. nana $(\mathrm{OR}=8.9$; IC95\%=1.2-66.1) y vivir al borde de acequias estuvo asociado a la infección con $A$. lumbricoides $(\mathrm{OR}=2.1$; IC95\%=1.3-3.2). La crianza de porcinos presentó más oportunidad de infectarse con Taenia $\mathrm{sp}(\mathrm{OR}=2.2$; IC95\%=1.2-4.2) y la crianza de pollos presentó más oportunidad de infectarse con $E$. vermicularis $(\mathrm{OR}=3.8$; IC95\%=1.4-10.1).

\section{Discusión}

La prevalencia de enteroparasitosis encontrada en este estudio ( $82.4 \%$ ) se encuentra dentro de las más elevadas a nivel nacional y del extranjero; lo cual se debería a la inadecuada higiene y saneamiento ambiental que existe en el sector rural, relacionada a factores culturales y socioeconómicos de la población, dominante humedad de los suelos areno-arcillosos y la tupida cobertura vegetal del terreno (Pajuelo et al., 2003; Aguin et al., 2011; Zonta et al., 2011). Es importante, además, considerar que la mayor parte de las viviendas tiene piso de tierra, lo cual puede constituir un factor relevante para el parasitismo de los niños (Giraldo-Ospina et al., 2015). Además, las enteroparasitosis son más frecuentes en poblaciones infantiles ante la insuficiente inmunidad existente propia de su edad (Albán et al., 2002).

La mayor prevalencia correspondió a Entamoeba coli $(59.6 \%)$, cuya presencia es indicador de una fuente común de contaminación, posiblemente a través del agua de bebida o del alimento; además de ser indicador de pobreza (Cabrera et al., 2000; Jiménez et al., 2011). Es producto de la contaminación con heces (Saredi, 2002), de los deficientes hábitos de higiene (Maco et al., 2002b) y la ausencia de desagües (Ibáñez et al., 2004; Jiménez et al., 2011). Asimismo, la ocurrencia de Taenia sp (33.3\%) tiene diversos factores en la zona que facilitan su transmisión debido al acceso de los animales a heces humanas (Staff et al., 2003; Owen, 2005).

Las cargas parasitarias de Enterobius vermicularis $(24.1 \%)$, Hymenolepis nana (19.4\%), Entamoeba coli (7.8\%) y Ascaris lumbricoides (1.6\%) encontradas en el estudio, son típicas de países en vías de desarrollo, especialmente de zonas rurales, donde el agua está altamente contaminada con materia fecal (Cabeza et al., 2015).

La prevalencia de protozoarios fue mayor a la de helmintos debido a que, los niños en sus instituciones educativas son eventualmente desparasitados en campañas del Centro de Salud del distrito usando generalmente albendazol, agente antihelmíntico recomendado para ser administrado bianualmente cuando la prevalencia basal es superior al 50\% (OMS, 2019). 
En el estudio, el estado nutricional predominante en los niños fue el normal (97.4). Sin embargo, la población escolar está cambiando sus estándares alimenticios y de actividad física, los cuales se asocian en algunos casos con sobre peso y obesidad, constituyéndose en una población con elevado riesgo (Zonta et al., 2011; Mispireta, 2012). Por otro lado, no se encontró asociación estadística significativa entre la parasitosis y la valoración nutricional antropométrica mediante el Índice de Masa Corporal en niños, situación similar a la observada en estudios realizados en Lima y en Venezuela (Borjas et al., 2009; Jiménez et al., 2011), posiblemente indicando que la causa de desnutrición de esas poblaciones sería multifactorial y que la parasitosis fue un factor contribuyente, pero no definitivo de la misma (Solano et al., 2008).

Ciertas características de las viviendas de los niños (sin suministro de agua, defecar a campo abierto, vivir al borde de cultivos y vivir al borde de acequias) estuvieron asociadas a $E$. vermicularis, $H$. nana y $A$. lumbricoides, por lo que la calidad de la vivienda está estrechamente relacionada con la salud de quienes la habitan (Marcos et al., 2002; Natividad y Terashima, 2008; Santa María, 2008; Rivera et al., 2010).

Aunque hay poca información sobre crianza de animales de traspatio en la región Apurímac; existen similares reportes a los de este estudio en Andahuaylas (Altamirano et al., 2014) y Abancay (Arando, 2017). En el distrito de Santa María de Chicmo la crianza de animales es familiar (autoconsumo); sin embargo, la crianza de porcinos y bovinos representa su principal fuente de ingreso. $\mathrm{La}$ crianza de animales sin el debido cuidado sanitario conlleva a un riego de importancia para la infestación de parásitos intestinales, no solo para los niños, sino para toda la familia (Devera et al., 2006; Leiva, 2012; O'Neal et al., 2012; Pham et al., 2013).

Se encontró asociación estadística significativa entre parasitosis y la crianza de porcinos y pollos en las viviendas de los ni- ños; por lo tanto, la convivencia con animales estaría asociada a la infección, principalmente en niños (Ccama y Sánchez, 1990; Carrada y Escamilla, 2005; Valencia et al., 2005). Taenia solium es de importancia en salud pública debido a que las personas pueden infectarse con huevos de la taenia y desarrollar cisticercos en sus tejidos.

\section{Conclusiones}

- La prevalencia de entoeroparasitosis en escolares del distrito de Santa María de Chicmo fue elevada.

- Los parásitos más prevalentes fueron Entamoeba coli, Ascaris lumbricoides y Taenia sp.

- La crianza de porcinos y pollos estuvo asociada a Taenia sp y E. vermicularis, respectivamente.

- Defecar a campo abierto, vivienda al borde de cultivos y al borde de acequias estuvieron asociados a E. vermicularis, H. nana y A. lumbricoides.

- El estado nutricional no estuvo asociado a la parasitosis en los escolares.

\section{Literatura Citada}

1. Aguilar L, Contreras M, Calle M. 2015. Guía técnica para la valoración nutricional antropométrica de la persona adolescente. Lima. Instituto Nacional de Salud.

2. Aguin V, Rivero AS, Sequera I, Serrano R, Pulgar V, Renzo I. 2011. Prevalencia y relación entre parasitosis gastrointestinal y bajo rendimiento académico en escolares que acuden a la escuela Bolivariana de Jayana, Falcon. Venezuela 2009. Revista CES Salud Pública 2(2).

3. Albán M, Jave J, Quispe T. 2002. Fasciolasis en Cajamarca. Rev Gastroenterol Perú 22:1.

4. Altamirano F, López R, Puray N. 2014. Enteroparásitos con potencial Zoonótico en Pacientes Pediátricos del 
Hospital de Andahuaylas - Apurímac. Salud Tecnol Vet 2: 14-19. doi: 10.20453/ stv.v2i1.1887

5. Arando JJ. 2017. Enteroparasitosis asociada a la crianza de animales domésticos y malas prácticas de higiene en niños de nivel primario del distrito de Tamburco. Tesis de Médico Veterinario Zootecnista. Apurímac, Perú: Univ. Nacional Micaela Bastidas de Apurímac. 80 p.

6. Beltrán M, Tello R, Náquira C. 2003. Manual de procedimientos de laboratorio para el diagnóstico de los parásitos intestinales del hombre. Serie de Normas Técnicas 37. Lima. Ministerio de Salud, [Internet].Disponible en:. https:// bvs.ins.gob.pe/ins print/ SALUD_PUBLICA/NOR_TEC/2014/ serie_normas_tecnicas_nro_37.pdf

7. Borjas P, Arenas F, Angulo Y. 2009. Enteroparasitismo en niños y su relación con la pobreza y estado nutricional. CIMEL 14: 49-54.

8. Cabeza MI, Cabezas MT, Cobo F, Salas J, Vázquez J. 2015. Hymenolepis nana: factores asociados a este parasitismo en un área de salud del Sur de España. Rev Chilena Infectol 32: 593595. doi: 10.4067/S0716-10182015000600019

9. Cabrera M, Verástegui M, Cabrera $R$. 2000. Prevalencia de enteroparasitosis en una comunidad altoandina de la Provincia de Víctor Fajardo, Ayacucho, Perú. Rev Gastroenterol Perú 25: 150-155.

10. Carrada T, Escamilla JR. 2005. Imágenes de patología clínica. Fasciolosis: revisión clínico-epidemiológica actualizada. Rev Mex Patol Clin 52: 283-296.

11. Ccama A, Sánchez C. 1990. Control integral de la distomatosis hepática en la irrigación Asillo. En: XI Congreso Nacional de Ciencias Veterinaria. Puno, Perú.

12. Devera $R$, Mago $Y$, Rumhein FA. 2006. Parasitosis intestinales y condiciones socio-sanitarias en niños de una comunidad rural del Estado Bolívar, Venezuela. Rev Biomed 17: 311-313.
13. Giraldo-Ospina B, Ramírez-Hoyos LS, Henao-Nieto DE, Flórez-Salazar M, Parra-Londoño F, Gómez-Giraldo EL, Mantilla-Moreno OJ. 2015. Estimación de la prevalencia de parásitos intestinales en niños de dos comunidades colombianas. Biosalud 14: 19-28. doi: 10.17151/biosa.2015.14.2.3

14. Ibáñez N, Jara C, Guerra A, Díaz E. 2004. Prevalencia del enteroparasitismo en escolares de comunidades nativas del Alto Marañón, Amazonas, Perú. Rev Peru Med Exp Salud Publica 21: 126-132.

15. Iannacone J, Benites MJ, Chirinos $L$. 2006. Prevalencia de infección por parásitos intestinales en escolares de primaria de Santiago de Surco, Lima, Perú. Parasitol Latinoam 61: 54-62. doi: 1.

16. [INEI] Instituto Nacional de Estadística e Informática. 2018. Perú en cifras. [Internet]. Disponible en: http:// www.inei.gob.pe/

17. Jiménez J, Vergel K, Velásquez M, Vega F, Uscata R, Romero S, Flórez $A$, et al. 2011. Parasitosis en niños en edad escolar: relación con el grado de nutrición y aprendizaje. Horizonte Médico 11: 65-69.

18. Leiva D. 2012. Antígenos del líquido pceudocelómico de Ascaris suum detectados por la técnica Western Blot utilizando IgC producidos en Oryctolagus cunniculus inmunizado experimentalmente. Tesis de Biólogo. Trujillo, Perú: Univ. Nacional de Trujillo. $30 \mathrm{p}$.

19. Maco V, Marcos L, Terashima A, Samalvides F, Miranda E, Espinoza J, Gotuzzo E. 2002a. Fas2-ELISA y la técnica de sedimentación rápida modificada por lumbreras en el diagnóstico de la infección por Fasciola hepatica. Rev Med Hered 13: 49-57.

20. Maco V, Marcos LA, Terashima A, Samalvides F, Gotuzzo E. 2002b. Distribución de la entereoparasitosis en el altiplano peruano: estudio en 6 comunidades rurales del departamento de Puno, Perú. Rev Gastroenterol Peru 22: 304-309 
21. Marcos LA, Maco V, Terashima A, Samalvides F, Gotuzzo E. 2002. Prevalencia de parasitosis intestinal en niños del valle del Mantaro, Jauja, Perú. Rev Med Hered 13: 85-89. doi: 10.20453/ rmh.v13i3.695

22. Mispireta ML. 2012. Determinantes del sobrepeso y la obesidad en niños en edad escolar en Perú. Rev Peru Med Exp Salud Pública 29: 361-365.

23. Natividad IS, Terashima A. 2008. Prevalencia de infección humana por Fasciola hepatica en pobladores del distrito de Caujul provincia de Oyon, región de Lima, Perú. Acta Med Per 25: 77-80.

24. [OMS] Organización Mundial de la Salud. 2019. Perú: Tratamiento vermífugo en niños. [Internet]. Disponible en: https://www.who.int/elena/titles/ deworming/es/

25. O'Neal SE, Moyano LM, Ayvar V, Gonzalvez G, Diaz A, Rodriguez S, Wilkins PP, Tsang VC, et al. 2012. Geographic correlation between tapeworm carriers and heavily infected cysticercotic pigs. PLoS Negl Trop Dis 6: e1953. doi: 10.1371/journal.pntd.0001953

26. Owen IL. Parasitic zoonoses in Papua New Guinea. J Helminthol 79: 1-14. doi: 10.1079/joh2004266

27. Pajuelo J, Rocca J, Gamarra M. 2003. Obesidad infantil: sus características antropométricas y bioquímicas. An Fac Med 64: 21-26.

28. Pham-Duc P, Nguyen-Viet H, Hattendorf J, Zinsstag J, Phung-Dac C, Zurbrïgg C, Odermatt P. 2013. Ascaris lumbricoides and Trichuris trichiura infections associated with wastewater and human excreta use in agriculture in Vietnam. Parasitol Int 62: 172-180. doi: 10.1016/j.parint.2012.12.007

29. Rivera M, Rodríguez C, Rojas Y, Valdivia Y, Saucedo T. 2010. Conocimientos, actitudes y prácticas sobre fascioliasis en madres de una zona rural andina del norte peruano. Rev Peru Med Exp Salud Pública 27: 59-62.

30. Sánchez EL, Náquira CG, Vega ES, Miranda EF, Quispe WM, Ayala ER. 2010. Manual de procedimientos para el diagnóstico serológico de las zoonosis parasitarias. Serie de Normas Técnicas 32. $2^{\circ}$ ed. Lima, Perú. Ministerio de Salud. $106 \mathrm{p}$.

31. Santa María R. 2008. La iniciativa de vivienda saludable en el Perú. Rev Peru Med Exp Salud Pública 25: 419-430.

32. Saredi NG. 2002. Manual práctico de parasitología médica. Buenos Aires, Argentina: Alfa Beta. $101 \mathrm{p}$.

33. [SENAMHI] Servicio Nacional de Meteorología e Hidrología del Perú. 2018. Perú: clima en el Perú. [Internet]. Disponible en: http://www.senamhi.gob.pe/

34. Solano L, Acuña I, Barón M, Morón A, Sánchez A. 2008. Influencia de las parasitosis intestinales y otros antecedentes infecciosos sobre el estado nutricional antropométrico de niños en situación de pobreza. Parasitol Latinan 63: 12-19. doi: 10.4067/S0717-77122008000100003

35. Staff PN, Acha B, Szyfres. 2003. Zoonoses and communicable diseases common to man and animals: In: Parasitoses. $3^{\text {rd }}$ ed. Washington DC; PAHO. $p$ 136-140.

36. Steinmann P, Usubalieva J, Imanalieva C, Minbaeva G, Stefiuk K, Jeandron A, Utzinger J. 2010. Rapid appraisal of human intestinal helminth infections among schoolchildren in Osh oblast, Kyrgyzstan. Acta Trop 116: 178184. doi: 10.1016/j.actatropica.2010.06.008

37. Uribe N, Sierra R, Espinosa C. 2012. Comparación de las técnicas Kato-Katz, TSET y TSR en el diagnóstico de infección por Fasciola hepática en humanos. Salud UIS 44: 7-12. 
38. Valencia $N$, Pariona A, Huamán M, Miranda F, Quintanilla S, Gonzáles A. 2005. Seroprevalencia de fasciolosis en escolares y en ganado vacuno en la provincia de Huancavelica, Perú. Rev Peru Med Exp Salud Pública 22: 96-102.
39. Zonta ML, Garraza M, Castro L, Navone GT, Oyhenart EE. 2011. Pobreza, estado nutricional y enteropasitosis infantil: un estudio transversal en Aristóbulo del Valle, Misiones, Argentina. Nutr Clín Diet Hosp 31: 48-57. 